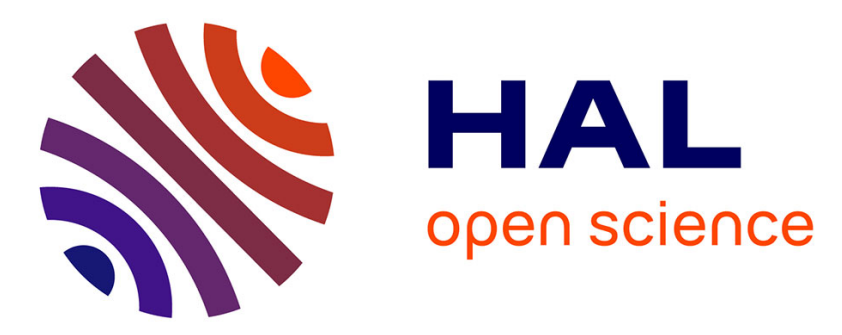

\title{
A feedback model of attentional effects in the visual cortex
}

\author{
Thomas Miconi, Rufin Vanrullen
}

\section{To cite this version:}

Thomas Miconi, Rufin Vanrullen. A feedback model of attentional effects in the visual cortex. IEEE Symp Computational Intelligence for Multimedia, Signal and Vision Processing (CIMSIVP 2011), Apr 2011, Paris, France. pp.106-113. hal-00706798

\section{HAL Id: hal-00706798 https://hal.science/hal-00706798}

Submitted on 11 Jun 2012

HAL is a multi-disciplinary open access archive for the deposit and dissemination of scientific research documents, whether they are published or not. The documents may come from teaching and research institutions in France or abroad, or from public or private research centers.
L'archive ouverte pluridisciplinaire HAL, est destinée au dépôt et à la diffusion de documents scientifiques de niveau recherche, publiés ou non, émanant des établissements d'enseignement et de recherche français ou étrangers, des laboratoires publics ou privés. 


\title{
A Feedback Model of Attentional Effects in the Visual Cortex
}

\author{
Thomas Miconi and Rufin VanRullen \\ Laboratoire Cerveau et Cognition (CerCo) \\ CNRS, UMR 5549 - Université Paul Sabatier \\ Toulouse, France \\ thomas.miconi@gmail.com
}

\begin{abstract}
Attention plays an important role in natural vision. Implementing realistic attentional processes in artificial vision systems could greatly improve their performance. However, existing models of attention do not adequately capture all of its complex effects on neural activity. In particular, existing models cannot reproduce recently reported effects such as shifting and scaling of receptive fields. Here we suggest that many of these effects arise naturally from feedback connections between visual areas (which redistribute top-down attentional modulation) and local, nonspecific short-range inhibition (which produce competition between stimuli that is automatically scaled to receptive field size). We show that a simple model with two reciprocally connected layers and shortrange inhibition can generate many known effects of attention, including receptive field shift and resizing. Due to its conceptual simplicity, the model may be easily integrated into a broad range of computer vision systems.
\end{abstract}

Keywords-attention; vision; feedback; inhibition

\section{INTRODUCTION}

\section{A. Attention has complex effects on neural responses}

State-of-the-art object recognition systems are still very far from competing with natural vision [1]. One possible explanation for this performance gap is that human object recognition makes use of attention. Attention allows for the selection of relevant parts of the visual input, facilitating recognition by downstream processes [2]. Therefore, a detailed understanding of attentional mechanisms could be highly valuable for improving the performance of computational vision systems.

Attention can modulate the activity of neurons in diverse ways. Very broadly, attention tends to increase the activity of neurons responding to attended stimuli or features, and reduce the activity of neurons responding to non-attended stimuli or features. Some studies suggest that attention results in a response gain (upward shift of the response curve) $[3,4]$, while others suggests an input/contrast gain (leftward shift of the response curve, without any increase in the maximum) [5]. Attention is also known to bias competition between different stimuli occurring within a given receptive field (RF), resulting in a response closer to that elicited by the attended stimulus alone [6]. Interestingly, the range of competition between stimuli is scaled to RF size
[2]: stimuli only compete (that is, simultaneous presentation of different stimuli produces a response intermediate between the responses elicited by either stimulus alone) if they both fall within the RF of the observed neuron, regardless of RF size. Besides these spatially-specific effects, attention can also be directed towards certain visual features, increasing the response of neurons preferring the attended feature over a broad spatial range, and resulting in an increase of selectivity at the population level [7].

How can these diverse results be explained by a single mechanism? One common proposal is to model attention as a localized excitatory modulation, interacting with lateral inhibition between neurons which normalizes activities over a local area $[6,8,9]$. An advantage of such models is that they are easily interpreted in terms of neural interactions: attentional modulation corresponds to excitatory input from higher areas, while normalization suggests mutual inhibition by neighboring inhibitory neurons. Recently, Reynolds and Heeger [8] proposed a simple model in which attention multiplies excitations, while inhibition normalizes the modulated responses - that is, a neuron's response is divided by the total excitation of surrounding neurons, plus a constant. They showed that this simple model could reproduce many of the effects of attention, including contrast gain, response gain, biased competition, and sharpening of tuning curves, depending on parameters such as the relative sizes of the attentional field and the attended stimulus.

However, recent studies have reported results that cannot be readily reproduced by such models: attention was shown to alter the position, size and structure of receptive fields (RF), generally shifting a neuron's RF towards the attended stimulus, both spatially and feature-wise. Womelsdorf et al. showed that attention shifts the RF of nearby neurons towards the focus of attention [10]. This confirmed previous results obtained by Connor and colleagues [11]. Additionally, Anton-Erxleben et al. [12] showed that directing attention within the RF of a given neuron will shrink this neuron's RF (in addition to shifting it), but directing it just outside the RF will instead extend the RF towards the focus of attention. David et al. [13] showed that attention can shift receptive fields not only spatially, but also feature-wise: the neuron's RF is altered to be more responsive to the type of feature 


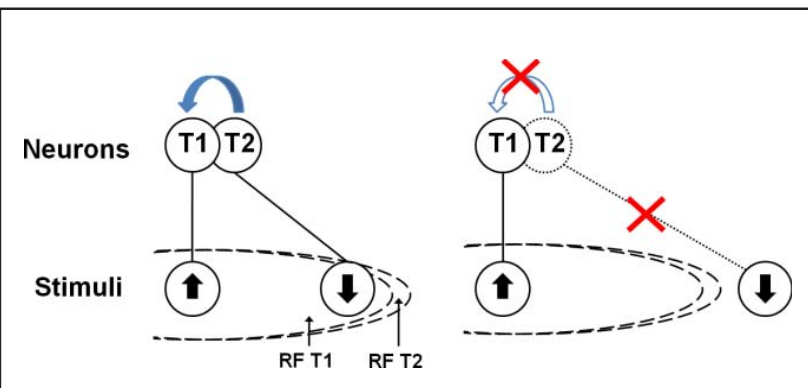

Figure 1. Short-range inhibition scales competition to RF size, because a neuron can only be inhibited by nearby neurons, which have very similar $\mathrm{RF}$. If an anti-preferred stimulus falls within the RF of neuron T1, it also falls within the RF of nearby neurons tuned to this stimulus (here T2), which respond to it and thereby inhibit T1 (Left). However, if the antipreferred stimulus falls well beyond T1's RF, it also falls beyond T2's

RF and cannot excite it. T1 will therefore not be inhibited (Right).

being attended to. This latter result is especially remarkable, given the complexity of receptive field structures and stimulus tuning of the neurons studied (in area V4, sensitive to features of intermediate complexity).

Simple modulations of neural responses cannot readily explain this effect: increasing or decreasing a neuron's response may push its entire response curve up or down, but cannot by itself shift it laterally, much less restructure it over fine feature space. Womelsdorf et al. [14] showed that spatial RF shifts and shrinks can be explained by multiplicativedivisive gains applied directly to a selected subset of the inputs of the relevant neurons; this had previously been suggested by McAdams and Maunsell [4] and by David et al. [13] However, this poses the question of how attention can reach these inputs directly, and especially how it "knows" which particular inputs to modulate in order to obtain the appropriate shift; again, the latter question is particularly acute for cells with complex RFs, which presumably involve fine-tuned connections to different input neurons within overlapping populations. Furthermore, attentional effects seem to proceed in a top-down direction, from higher to lower areas, rather than the opposite $[15,16]$.

Compte and Wang [17] have suggested that these RF shifts could be explained by lateral connections within the studied layer. However, their model assumes a fixed "Mexican Hat" connectivity pattern in which short-range connections are excitatory while long-range connections are inhibitory, while in reality the sign of lateral interactions actually varies with stimulus intensity (a same stimulus in the surround will increase response to a weak central stimulus, but decrease response to a strong central stimulus [18]). Perhaps more importantly, the model of Compte and Wang does not readily explain how attention can extend a neuron's RF of nearby neurons when it is located just outside this $\mathrm{RF}$ rather than inside it, as reported by Anton-Erxleben et al [12].

Additionally, Compte and Wang's model assumes that lateral inhibition is exerted directly through lateral connections. This does not explain why the range of lateral competitive effect is scaled to the size of the RF, across areas, even as RF size varies across areas; rather, it simply assumes strong lateral inhibition that happens to be tuned to RF size. This latter limitation is also shared by Reynolds and Heeger's model, in which competition occurs through mutual inhibition within a layer. This implies that the range of lateral inhibition changes greatly from one area to the next, precisely matching RF size in every area. While not impossible a priori, this represents a significant assumption.

\section{B. A simple model of attention: feedback + local inhibition}

Here we suggest that all the effects of attention described above (response or input gain, biased competition scaled to RF size, feature-similarity gain, spatial and feature-wise RF shifting, shrinking and extension) can be simply explained by considering the role of feedback connections in redistributing activity between areas. We argue that in a multi-layer system, the action of a top-down multiplicative modulation, applied to the topmost layer, propagating through feedback connections from layer to layer, and interacting with local, short-range, nonspecific inhibition, suffices to recreate all the effects discussed above. The diverse consequences of this simple model can be understood intuitively by noticing two effects:

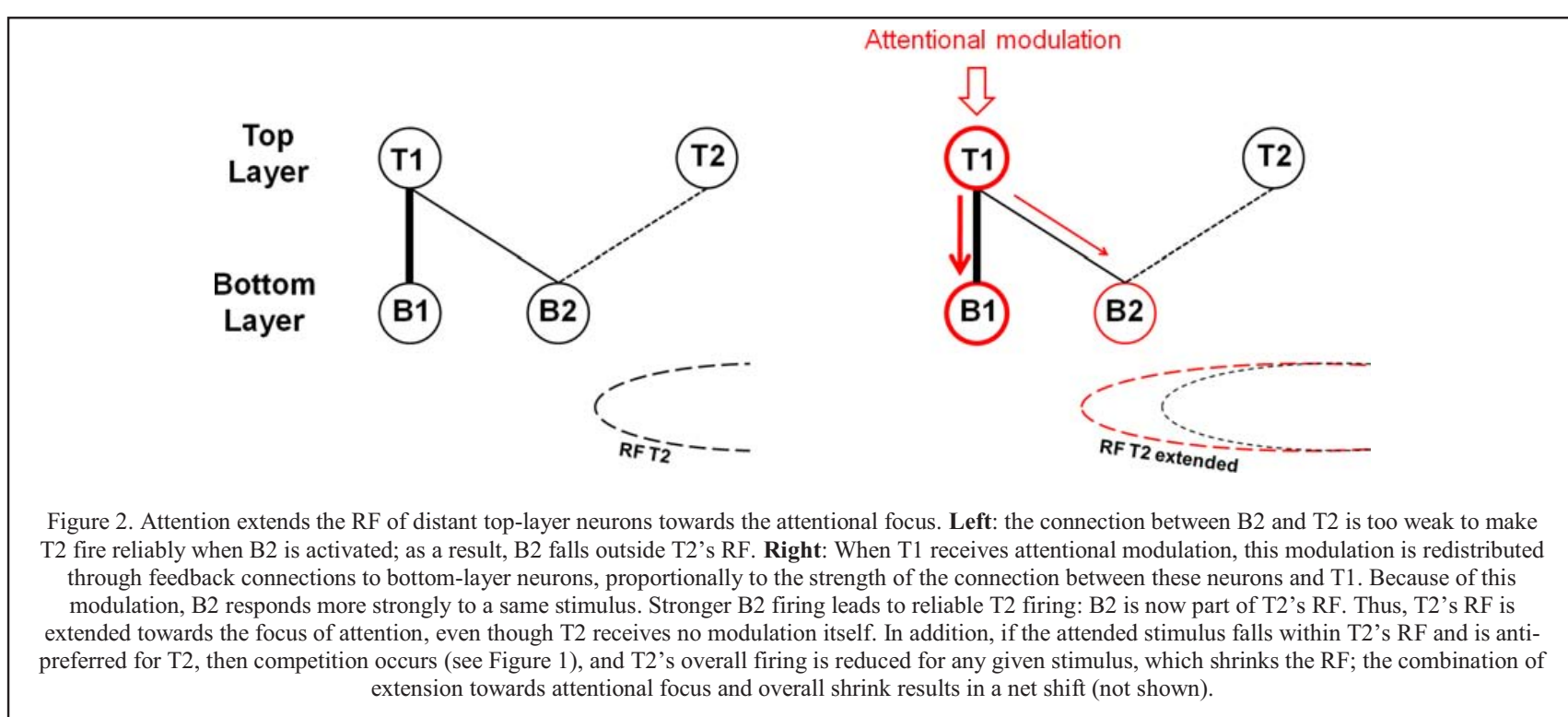




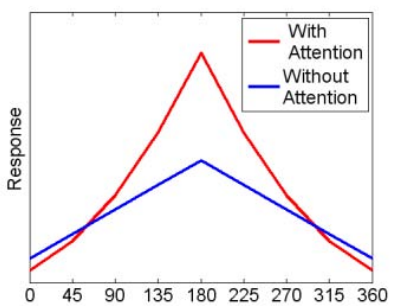

A

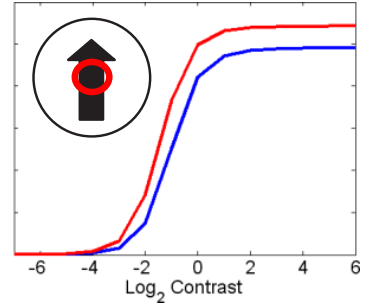

B

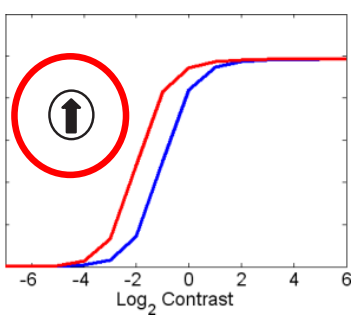

C

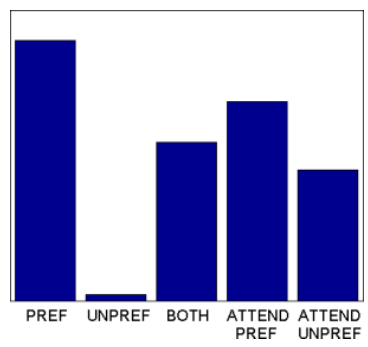

D

Figure 3: Replication of previous results [8]. A) Stimuli of varying orientations are presented to a cell tuned to respond optimally to stimuli oriented at 180 degrees. In the "with attention" condition, attention is drawn to the orientation of the presented stimulus. Feature-based attention results in a sharpening of the tuning curve. B) A small attentional field over a large stimulus results in a response gain. C) A large attentional field over a small stimulus results in a contrast (input) gain. D) Biased competition: presenting both a preferred stimulus (PREF) and an anti-preferred stimulus (UNPREF) results in an intermediate response (BOTH), indicating competition between stimuli. Attending to either stimulus brings the response closer to that elicited by the stimulus alone, indicating that attention biases the competition between stimuli.

1- Short-range inhibition automatically scales the range of competition to the size of receptive fields - that is, with short-range inhibition, an inhibiting stimulus will automatically inhibit a top-layer neuron if it falls within its $\mathrm{RF}$, but not if it falls well outside, independently of RF size. This is because a top-layer neuron $\mathrm{N}$ is not directly inhibited by bottom-layer neurons (inter-areal connections are generally excitatory), but indirectly by neighboring neurons within the top-layer that are tuned to this anti-preferred stimulus (or broadly tuned to any stimulus), respond strongly to it, and thereby inhibit N. This can only happen if the oppositely tuned neighbors can also see the stimulus, which requires that the stimulus lies in their own RF. Since these neighbors must be very close to $\mathrm{N}$ (if they are to inhibit it using short-range inhibition), it follows that their RF is almost identical to N's (due to the retinotopic organization of visual cortex). Therefore the inhibiting stimulus must fall within N's RF (plus a small border corresponding to the short range of inhibition) to inhibit it (see Figure 1).

2- If we assume that connections between layers are reciprocal (i.e. identical feedforward and feedback connection patterns), an excitatory modulation applied to a neuron $\mathrm{N}$ in the top layer will be automatically redistributed, by feedback connections, to the very same neurons in the bottom layer that provide input to $\mathrm{N}$ - that is, to the neurons that compose its RF - and in proportion to how much they contribute to this RF. Due to this increase in stimulation, these modulated input-layer neurons will then affect the activity of all other top-layer neurons that have them within their own RF - even those that did not receive any direct attentional modulation: a stimulus falling upon these input neurons will produce a stronger response (because of the modulation), resulting in an extension of the top-layer RFs towards these inputs, both spatially and feature-wise. Importantly, notice that this effect can reach much further than the radius of a single RF, since all inputs composing N's $\mathrm{RF}$ will be modulated, and thus in turn will affect all neurons whose RF intersects with N's (see Figure 2).

We argue that, due to the combination of these two effects, a simple model including top-down excitatory modulation originating from attentional control areas (not modeled), short-range inhibition, and feedback between layers, can explain all the effects of attention discussed above. To demonstrate this, we build a simple numerical model including two areas linked by reciprocal connections, with local mutual inhibition. We demonstrate that this simple model reproduces all the previously mentioned effects of attention, and particularly the RF shift, shrink and extension that cannot be adequately reproduced by existing models.

\section{MODEL DESCRIPTION}

Our model is composed of two 2D layers linked by reciprocal connections. The cells in both layers are orientation-sensitive, with each cell preferring one of 8 possible discrete orientations between 0 and $7 \pi / 4$ inclusive. All results reported below are for the top layer, which is the layer of interest.

Bottom-layer cells receive direct input from the stimulus. Each pixel in the input picture is covered by 8 cells of the bottom layer - one for each possible orientation. The top layer also has 8 cells (one for each possible orientation) per pixel. Thus, both the bottom and top layer can be seen as bundles of 8 orientation maps, with reciprocal connections between the two.

Bottom-layer cells send driving connections to top-layer cells, with the strength of the connection depending both on the distance between the cells and the difference between their preferred orientations. More precisely, the weight of a connection between bottom-layer cell $\mathrm{i}$ and top-layer cell $\mathrm{j}$, with respective positions $\mathrm{x}_{\mathrm{i}}, \mathrm{x}_{\mathrm{j}}$ and preferred orientations $\theta_{\mathrm{i}}$, $\theta_{\mathrm{j}}$ is given by the following equation:

$$
\begin{aligned}
\mathrm{W}(\mathrm{i}, \mathrm{j}) & =\mathrm{W}_{\max } * \exp \left(-\left(\mathrm{x}_{\mathrm{i}}-\mathrm{x}_{\mathrm{j}}\right)^{2} /\left(2 * \mathrm{R}_{\mathrm{RF}}{ }^{2}\right)\right) *\left(1-4 * \operatorname{Dist}\left(\theta_{\mathrm{i}},\right.\right. \\
\left.\left.\theta_{\mathrm{j}}\right) / 4.1 \pi\right) & (1)
\end{aligned}
$$

where $\operatorname{Dist}\left(\theta_{\mathrm{i}}, \theta_{\mathrm{j}}\right)$ is the angular distance between angles $\theta_{\mathrm{i}}$ and $\theta_{\mathrm{j}}$. taking values between 0 and $\pi$; because there are only 8 discrete possible orientations, $4 * \operatorname{Dist}\left(\theta_{\mathrm{i}}, \theta_{\mathrm{j}}\right) / \pi$ takes integer values between 0 and 4 inclusive.

Intuitively, this equation models a receptive field as a Gaussian function centered on $\mathrm{x}_{\mathrm{i}}$, with standard deviation $\mathrm{R}_{\mathrm{RF}}$ (indicating the "radius" of the receptive field), and multiplied by a factor that decreases linearly (from 1 to almost zero) with the angular distance between the preferred orientations of cells $\mathrm{i}$ and $\mathrm{j}$.

Similarly, top-layer cells send modulatory feedback connections to bottom-layer cells. The equation for the 


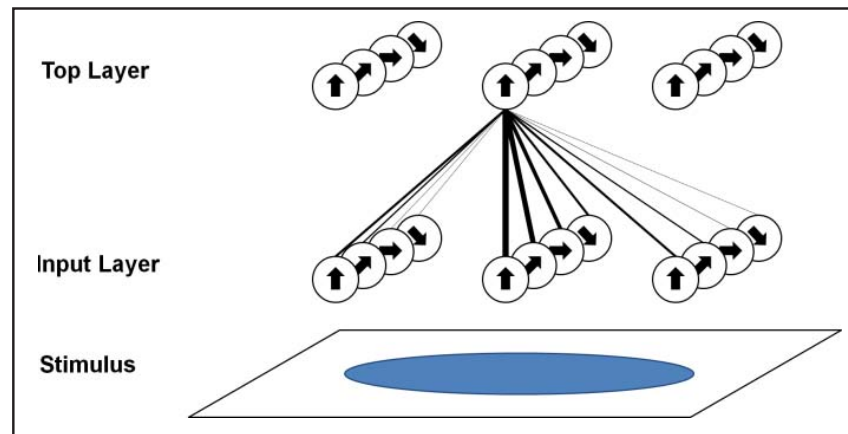

Figure 4: Schematic description of the model. At each pixel, both layers have a full complement of 8 cells sensitive to different orientations (only 4 directions are shown here for clarity). Top-layer cell are reciprocally connected to bottom-layer cells; the strength of the connection decreases with the distance between their receptive fields, as well as the difference between their preferred orientations. See text for details.

weight of feedback connections is the same as the one for feedforward connections above; however, while feedforward excitation constitutes the actual input of top-layer cells, feedback excitation, being modulatory, merely multiplies the sensory input to bottom-layer cells. Thus, feedback excitation alone is insufficient to make a cell fire significantly, but enhance its response if it is already firing, in accordance with observations and theory [19].

Attentional modulation is modelled as a pointwise multiplication of top-layer cell excitations by one plus a Gaussian function centered at the focus of attention, with standard deviation $\mathrm{R}_{\text {Att }}$. However, some simulations involve feature-based attention rather than spatial attention. In this case, attention is instead implemented as a multiplication of each cell i's excitation by $1-4 * \operatorname{Dist}\left(\theta_{\mathrm{i}}, \theta_{\text {Att }}\right) / 4.1 \pi$, where $\theta_{\text {Att }}$ is the attended direction, and $\operatorname{Dist}\left(\theta_{\mathrm{i}}, \theta_{\text {Att }}\right)$ is again the angular distance between $\theta_{\mathrm{i}}$ and $\theta_{\mathrm{Att}}$.

Together, bottom-layer input and attentional modulation define the excitation of a cell. The actual response of a cell is also affected by inhibition. Following Reynolds and Heeger's normalization model of attention [8], local inhibition is modelled as a pointwise division of top-layer excitations (including attentional modulation) by the average activity of their immediate neighbourhood, plus a constant $\sigma$. This average local excitation is obtained for each point by convolving top-layer activities with a Gaussian filter (with standard deviation $\mathrm{R}_{\text {Inh }}<<\mathrm{R}_{\text {Att }}<<\mathrm{R}_{\mathrm{RF}}$ ) for all orientation maps, then taking the average over all orientations for each point.

Thus, the response of a top-layer cell can be written as

$$
\mathrm{R}(\mathrm{x})=\mathrm{A}(\mathrm{x}) * \mathrm{I}(\mathrm{x}) /(\mathrm{S}(\mathrm{x})+\sigma),
$$

where $\mathrm{I}(\mathrm{x})$ is the input received by this cell from bottomlayer cells, $\mathrm{A}(\mathrm{x})$ is the value of the attentional modulation at this point, $\mathrm{S}(\mathrm{x})$ is the value of the "suppression field" (the inhibitory input calculated by averaging local excitations, inclusive of attentional modulation), and $\sigma$ is a constant that determines the semi-saturation level of excitation (following Reynolds and Heeger [8]).
The resulting top-layer activity is then used to multiply the activity of bottom-layer cells, as specified by the weights calculated from Equation (1). Because the bottom-layer activities have changed, the top-layer activities (which use bottom-layer activities as their inputs) must be re-calculated. The entire process is then iterated three times (simulations with 20 iterations did not produce qualitatively different results).

\section{RESULTS}

\section{A. Reproduction of previous results}

As a safety check, we first reproduce the most salient results reported with existing models of attention, especially Reynolds and Heeger's normalization model [8]. In particular, we show that our own model reproduces the shift from response gain (multiplication of the response) to contrast gain (multiplication of the input) depending on the relative sizes of stimuli and attentional field. We also reproduce the sharpening of tuning curves brought about by feature-based attention, in conformity with the featuresimilarity gain principle (Figure $3 b-d$ ).

We also sought to reproduce the effects of biased competition. In line with similar experiments [6], we put two stimuli of opposite directions within the receptive field of a given cell which prefers one of these orientations. We first present each stimulus alone, then both stimuli simultaneously. As expected, the response to simultaneous presentation is intermediate between the responses to both stimuli presented in isolation (Figure 3a). This demonstrates that our model produces long-range competition, despite only implementing short-range mutual inhibition.

We then introduce attention in the system, focusing attention upon each stimulus in turn; that is, attentional modulation is applied to the top layer at the spot that lies just over the stimulus (see Material and Methods). As expected, the resulting response from the observed neuron (which does not receive any direct attentional modulation itself) is brought closer to that elicited by the attended stimulus in isolation (Figure 3a). This is because the attentional modulation upon the top-layer cells is transmitted to the inputs over the attended stimulus, increasing their activity. This in turn increases the activity of either the observed neuron (if the stimulus is of the preferred orientation), or neighbouring, anti-oriented neurons (if the stimulus is of the anti-preferred orientation) relative to each other, allowing them to prevail in the competition over their neighbours imposed by normalization (see also Fig. 1).

\section{B. RF shift, shrink and extension.}

The results presented above demonstrate that our model can reproduce the effects of existing models; this is encouraging, but not really surprising. However, existing models cannot explain the shift, shrink and extension of RF induced by attention. We will now demonstrate that our own model, due to feedback interactions, reproduces these effects. 
To observe the effects of attention on RF structure, we implement experimental settings similar to those used in the original experiments by the Treue group $[10,12]$. We place two identical stimuli on the input field. Top-layer cells lying just midway between these two stimuli have them both in their RF, but other cells in the field may have only one or none of these stimuli within their RF. We focus attentional modulation on either of these two stimuli (i.e. on top-layer cells with RF centered on the stimulus), and then record the response of various cells as a probe stimulus is moved over the field. By mapping the response of a cell to a probe stimulus presented at various positions, we obtain a picture of its effective RF. Following original experiments [7, 9], we report results from cells whose orientation tuning is optimal for the probe, while the attended stimuli are of opposite orientation to the probe.
First, we study the effects of attention upon a cell lying midway between both stimuli. As expected, we show that switching attention from one stimulus to the other shifts the RF of this midway cell towards the attended stimulus (Figure 5, top row).

We then examine the effects of focusing attention within the RF, or just outside the RF. We first observe the activity of a cell situated just below one of the stimuli, such that this stimulus lies well within the RF of this cell. As shown on Figure 5 (middle row), focusing attention on this within-RF, anti-preferred stimulus produces both a shift and a shrink of the RF (in comparison to a no-attention situation). The response is increased over a portion of the field close to the attended stimulus, but decreased over a larger portion of the field further away from the stimulus, resulting in both a shift and a net shrink.
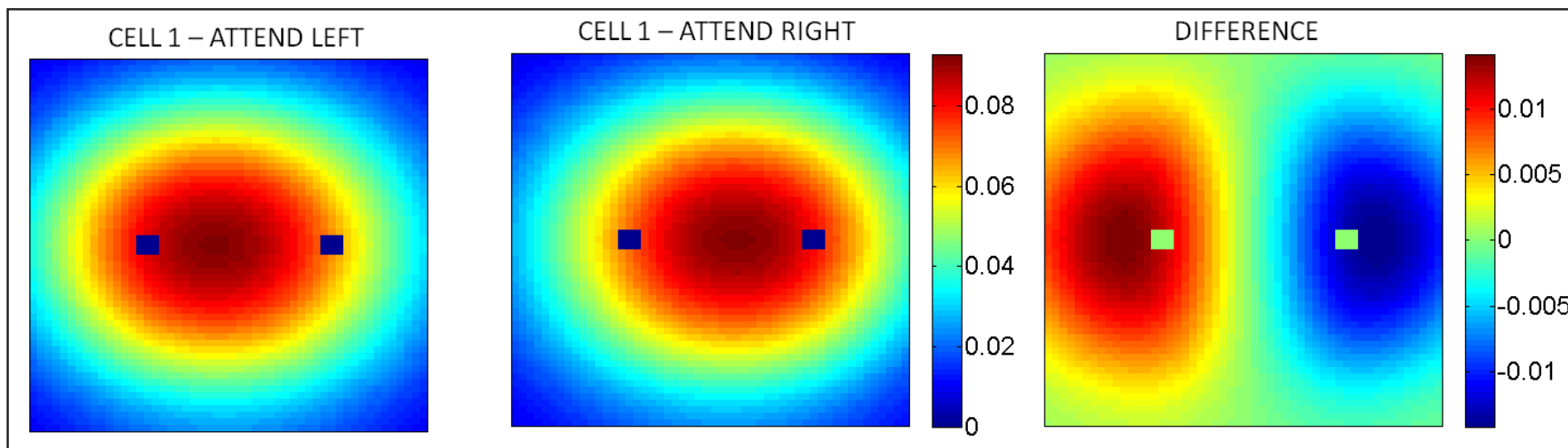

CELL 2 - NO ATTENTION

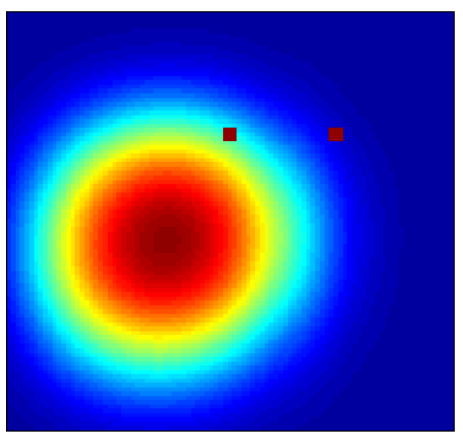

CELL 3 - NO ATTENTION

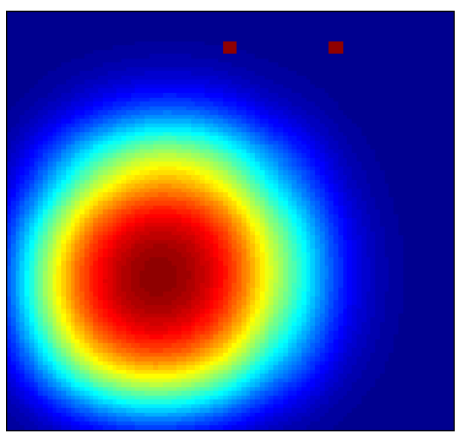

CELL 2 - ATTEND LEFT

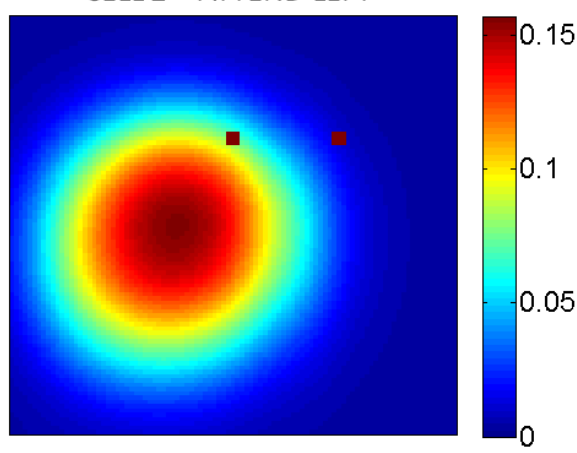

CELL 3 - ATTEND LEFT
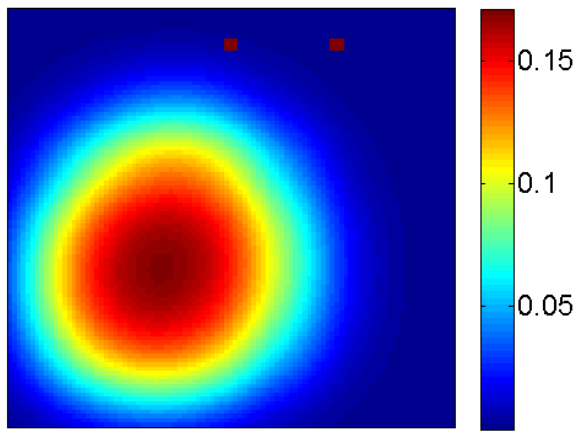

\section{DIFFERENCE}

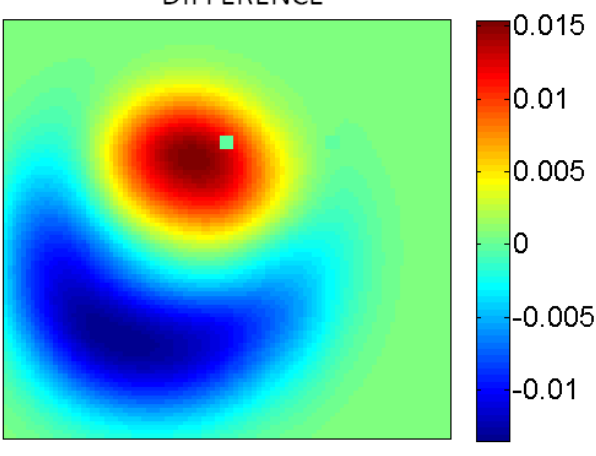

DIFFERENCE

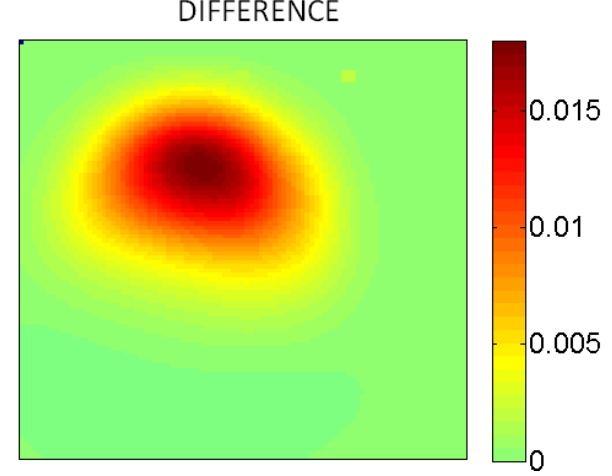

Figure 5. Top row: shifting attention on either side of a central cell shifts the receptive field of this cell towards this side. Middle row: Focusing attention on an antipreferred stimulus within a cell's RF will produce a small extension of the RF close to the focus of attention, and a large decrease in the RF away from it, resulting in an overall shift and shrink. Bottom row: focusing attention on an anti-preferred stimulus outside a cell's RF will produce an extension of this RF towards the focus of attention. These results are in full agreement with experimental observations. 


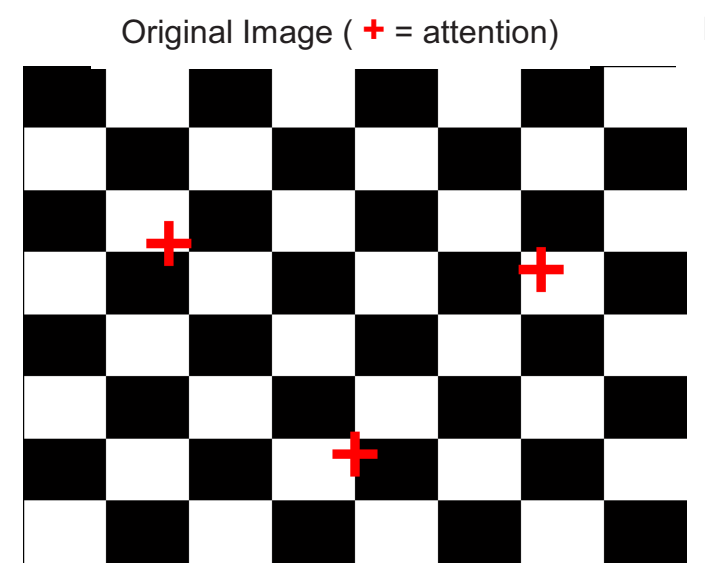

Reconstruction from top layer output (with attention)

Difference in reconstruction

(with attention - without attention)
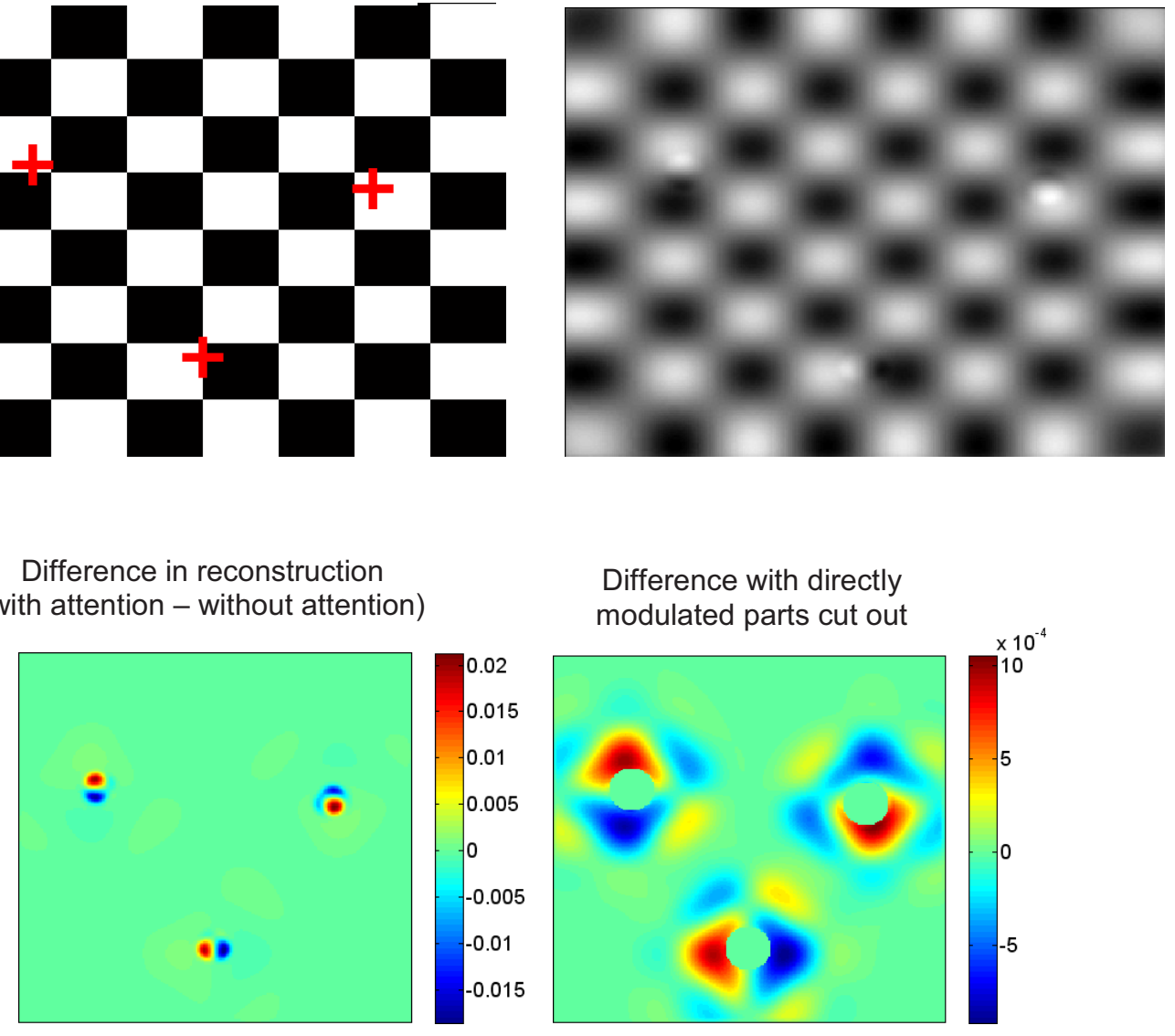

Difference with directly modulated parts cut out

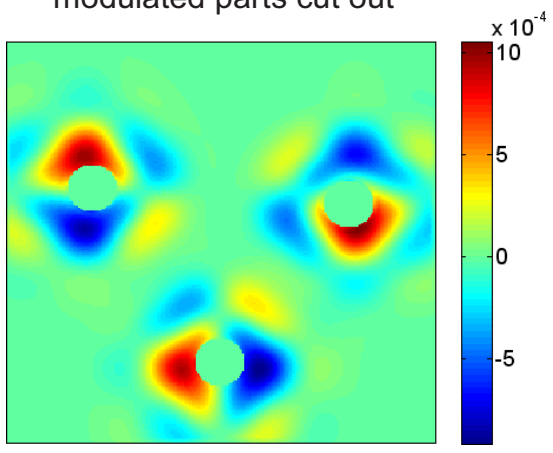

Abs(Reconstruction + 100*Difference)

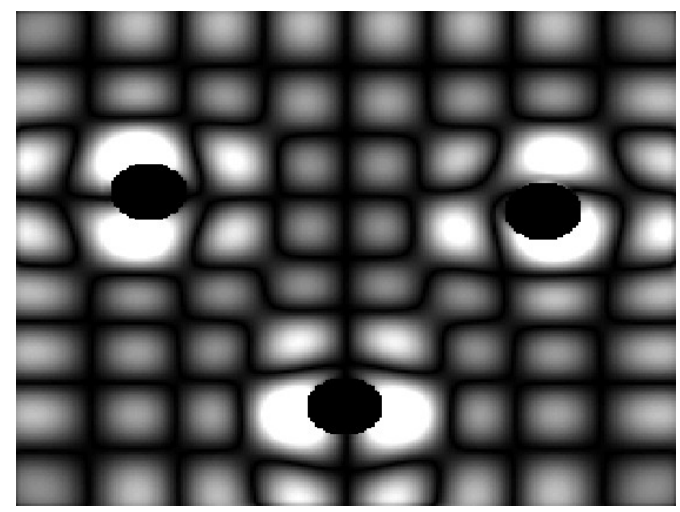

Abs(Reconstruction $+100 *$ Difference $)$, without feedback

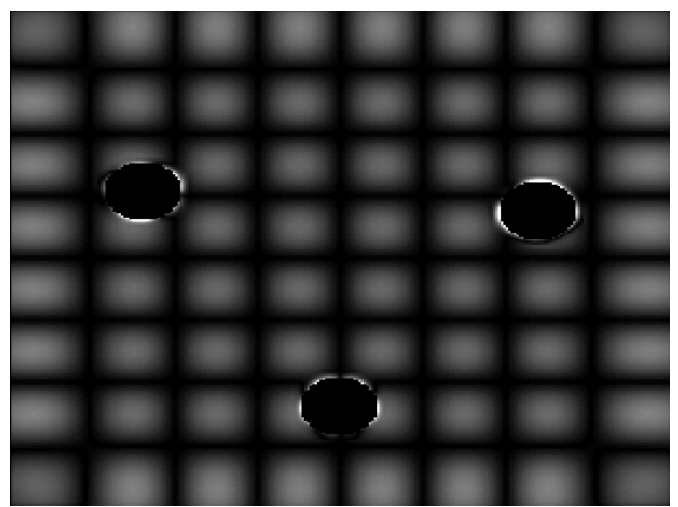

Figure 6. Results of the model using an actual image as input. The input image is a simple checkerboard; attention is focused on three different points within the image (indicated by red crosses; note that we do not assume that attention can simultaneously focus on 3 different locations --the point is merely to illustrate 3

distinct simulations on a single picture). The effects of attention upon top layer activity are relatively small (top-right) and are dominated by contrast gain imparted by direct attentional modulation (middle-left). However, if the zones that receive direct attentional modulation are cut out, complex effects ranging far from the directly modulated zones can be seen (middle-right). If we enhance the difference caused by attention on reconstruction from top-layer activity (taking absolute value of the results to increase clarity and emphasize zero-crossings), these long-distance effects turn out to produce a "zoom" around the focus of attention (bottom-left). If feedback connections are removed from the system, all long-distance effects disappear, confirming that these effects are brought about by feedback connections (bottom-right). 
By contrast, when studying a cell lying further from the attended stimulus, such that the stimulus lies just outside the RF of the cell (Figure 5, bottom row), we see that attending to the stimulus produces an extension of the field towards the focus of attention, rather than a pure shift or shrink. This is in full agreement with experimental results [12].

The alteration of RF structure is easily explained in our model. When attending to one of the stimuli, top-layer cells at the focus of attention receive a positive modulation, increasing their excitation. The added excitation on top-layer cells is then redistributed, through feedback connections, as a positive modulation upon input cells that constitute their RF. As a result, probe stimuli falling upon these input cells will produce a stronger response than they would have in the absence of attention, in turn generating a stronger response from all other top-layer cells that also have these input cells within their own RF, when the probe passes over this area. This results in an extension of the top-layer cells' RF towards the focus of attention, even if these top-layer cells do not receive any direct attentional modulation themselves (see Figure 2).

In addition, if the focus of attention lies within the RF of the observed cell, then biased competition occurs between the probe and the attended stimulus. Because the attended stimulus is anti-preferred for the observed cells (following the paradigm of the original experiments $[10,12]$ ), we expect that attending to the stimulus will reduce their overall activity. The combination of these two effects (extension of the RF towards the focus of attention, plus reduction of activity resulting in a shrink of the RF) together result in a shift and shrink of the cell's RF. By contrast, if the attended stimulus lies outside the RF of the cell, then competition does not occur and only the extension (caused by the modulated inputs) remains.

\section{APPLICATION TO IMAGES: ATTENTIONAL ZOOM}

In the previous sections we studied single-cell behavior, in order to compare the outcome of our model with experimental results. We now seek to explore the behavior of our model at the population level. In particular, we want to know how our model processes actual images, and what difference (if any) the mechanisms discussed above make in this processing.

For this purpose, we modify the input layer of our model in order to accommodate real image input. Each cell in the bottom layer now acts as a local filter, implementing oriented edge detection over a $5 \times 5$ window of the input picture. Each input pixel is associated with a full set of 8 cells (one for each orientation from 0 to 315 degrees in steps of 45 degrees), thus leading to high overlap between the receptive fields of neighboring cells. In all other respects, the model operates exactly as described above.

As a test image, we use a simple picture of a checkerboard (Figure 6 top-left). We chose a checkerboard picture because we expected that its regular geometry would highlight structural effects of the model. After running the model in the manner described in Section II, we reconstruct the image from the response of the model's top layer, in order to assess how the model "perceives" the picture. This is done simply by convolving each orientation map in the top layer with the appropriate $5 \times 5$ edge-detector filters of the same orientation as the map, then summing the results over all 8 maps for each pixel. Unsurprisingly, the result is rather blurry, since each top-layer cell samples input over a relatively wide area of the image. Nevertheless, the original structure is easily identifiable (Figure 6, top-right).

As can be seen from Figure 6 (top-right), the impact of attention on image processing is relatively small; furthermore, most of the effect consists in a highly localized contrast gain, essentially limited to the zone of attentional modulation. This effect is not surprising, considering that attentional modulation is multiplicative in our model; similar results would be expected with other multiplicative models, such as the standard normalization model [8].

However, if we remove the portions of the field directly modulated by attention (by cutting out the portion of the image where attentional modulation is above $1 \%$ of its maximum), we see that small attentional effects actually propagate quite far from the focus of attention (Figure 6 middle-right). This effect could not be seen in the raw difference map (Figure 6 middle-left), because it was overwhelmed by the much larger, localized effect of direct attentional modulation. In order to visualize the impact of these long-distance effects on image processing, we artificially enhance the impact of attention. To do this, we simply take the difference between the reconstructed images obtained with and without attention (after cutting out parts directly affected by attentional modulation), multiply this difference by a high constant, then add it to the no-attention reconstruction. The resulting "enhanced" reconstruction is shown in Figure 6, bottom-left (the absolute value is taken to emphasize geometrical effects, especially zero-crossings which appear as dark stripes). It reveals that the longdistance effect effectively behaves not just as a multiplicative modulation on contrast, but also as a geometrical "zoom", such that zones close to the focus of attention occupy a larger portion of the image.

It is important to note that this "zooming" effect is actually very small, which is why a highly enhanced reconstruction was needed to observe it. Nevertheless, the effect is in agreement with the experimental observation that attention shifts the receptive fields of individual neurons towards the attentional focus; hence, more neurons are recruited to encode the same portion of visual space around the focus, resulting in a "zoomed-in" representation. The findings also suggest that the single-cell behavior described in section III does produce a small, but coherent effect at the population level - and may therefore affect further perceptual processing by downstream areas.

\section{CONCLUSIONS}

In this paper we suggest that the complex effects of attention on neural activity within the visual system can be adequately explained by a top-down excitatory modulation, propagated through feedback connections, and interacting with local inhibition. The main contribution of this paper is 
to show that recently reported effects (namely the shifting and scaling of RF by attention), which cannot be reproduced by existing models, arise naturally once the effects of reciprocal connections between layers are taken into account. The conceptual simplicity of our model implies that it could be readily implemented in a broad range of artificial vision systems.

Importantly, the present model is only concerned with the effects of attention within the visual cortex itself. It does not cover the selection of the attentional target; rather, it assumes that this choice has already been made, and reified as a localized top-down modulation falling upon the topmost layers of the pure visual system (V4 or MT). In the brain, prime candidates for the source of this attentional "spotlight" are the lateral intraparietal cortex (LIP) and the frontal eye field (FEF). Both areas are known to be massively interconnected, leading to the concept of a "frontoparietal network" [2]. Some models attempt to explain how visual information is integrated to create a "salience map", the maximum of which determines where attention is to be allocated [20]. Combining these models with the one discussed here could provide a complete mechanistic model of visual attention.

\section{REFERENCES}

[1] N. Pinto, D. D. Cox, and J. J. Dicarlo, "Why is Real-World Visual Object Recognition Hard?," PLoS Comput Biol, vol. 4, p. e27, 2008.

[2] S. Kastner and L. G. Ungerleider, "Mechanisms of visual attention in the human cortex," Annu Rev Neurosci, vol. 23, pp. 315-41, 2000.

[3] S. Treue and J. C. Martinez Trujillo, "Featurebased attention influences motion processing gain in macaque visual cortex," Nature, vol. 399, pp. 575-9, 1999.

[4] C. J. McAdams and J. H. R. Maunsell, "Effects of attention on orientation-tuning functions of single neurons in macaque cortical area V4," J Neurosci, vol. 19, pp. 431-41, 1999.

[5] J. H. Reynolds, T. Pasternak, and R. Desimone, "Attention increases sensitivity of V4 neurons," Neuron, vol. 26, pp. 703-14., 2000.

[6] J. H. Reynolds, L. Chelazzi, and R. Desimone, "Competitive mechanisms subserve attention in macaque areas V2 and V4," J Neurosci, vol. 19, pp. 1736-53, 1999.

[7] J. C. Martinez-Trujillo and S. Treue, "FeatureBased Attention Increases the Selectivity of Population Responses in Primate Visual Cortex," Curr Biol, vol. 14, pp. 744-751, 2004.

[8] J. H. Reynolds and D. J. Heeger, "The normalization model of attention," Neuron, vol. 61, pp. 168-85, 2009.

[9] G. M. Boynton, "A framework for describing the effects of attention on visual responses," Vision Res, vol. 49, pp. 1129-43, 2009.
[10] T. Womelsdorf, K. Anton-Erxleben, F. Pieper, and S. Treue, "Dynamic shifts of visual receptive fields in cortical area MT by spatial attention," Nat Neurosci, vol. 9, pp. 1156-60, 2006.

[11] C. E. Connor, D. C. Preddie, J. L. Gallant, and D. C. Van Essen, "Spatial attention effects in macaque area V4," J Neurosci, vol. 17, pp. 3201-14, 1997.

[12] K. Anton-Erxleben, V. M. Stephan, and S. Treue, "Attention reshapes center-surround receptive field structure in macaque cortical area MT," Cereb Cortex, vol. 19, pp. 2466-78, 2009.

[13] S. V. David, B. Y. Hayden, J. A. Mazer, and J. L. Gallant, "Attention to stimulus features shifts spectral tuning of V4 neurons during natural vision," Neuron, vol. 59, pp. 509-21, 2008.

[14] T. Womelsdorf, K. Anton-Erxleben, and S. Treue, "Receptive Field Shift and Shrinkage in Macaque Middle Temporal Area through Attentional Gain Modulation," J Neurosci, vol. 28, pp. 8934-8944, 2008.

[15] E. A. Buffalo, P. Fries, R. Landman, H. Liang, and R. Desimone, "A backward progression of attentional effects in the ventral stream," Proc Natl Acad Sci US A, vol. 107, pp. 361-365, 2010.

[16] G. G. Gregoriou, S. J. Gotts, H. Zhou, and R. Desimone, "High-frequency, long-range coupling between prefrontal and visual cortex during attention," Science, vol. 324, pp. 1207-10, 2009.

[17] A. Compte and X. J. Wang, "Tuning curve shift by attention modulation in cortical neurons: a computational study of its mechanisms," Cereb Cortex, vol. 16, pp. 761-78, 2006.

[18] D. C. Somers, E. V. Todorov, A. G. Siapas, L. J. Toth, D. S. Kim, and M. Sur, "A local circuit approach to understanding integration of longrange inputs in primary visual cortex," Cereb Cortex, vol. 8, pp. 204-17., 1998.

[19] F. Crick and C. Koch, "Constraints on cortical and thalamic projections: the no-strong-loops hypothesis," Nature, vol. 391, pp. 245-50., 1998.

[20] L. Itti and C. Koch, "A saliency-based search mechanism for overt and covert shifts of visual attention," Vision Res, vol. 40, pp. 1489-506, 2000. 